\title{
SEGURANÇA CIRÚRGICA NA CESÁREA: REVISÃO INTEGRATIVA
}

Lara Mabelle Milfont Boeckmann¹, Maria Cristina Soares Rodrigues²

${ }^{1}$ Enfermeira. Doutoranda em Enfermagem. Universidade de Brasília. Brasília, DF, Brasil.

${ }^{2}$ Enfermeira. Pós-doutora em Ciências da Saúde. Docente do Departamento de Enfermagem. Universidade de Brasília. Brasília, DF, Brasil.

RESUMO: Objetivou-se reunir e analisar publicações científicas sobre segurança cirúrgica na cesárea com a seguinte questão: quais as evidências científicas sobre segurança cirúrgica na cesárea nos últimos dez anos? Realizou-se revisão integrativa da literatura, em sete bases eletrônicas, de outubro a dezembro de 2014: The US National Library of Medicine, Cumulative Index to Nursing and Allied Health Literature, Literatura Latino-Americana e do Caribe em Ciências da Saúde, Medical Literature analysis and Retrievel System Online, Cochrane Library, Base de dados em Enfermagem e Scientific Eletronic Library Online. Foram encontrados 463 artigos, dos quais 11 foram selecionados para análise, emergindo dois eixos temáticos: promoção da segurança da paciente na cesárea por meio da lista de verificação de segurança cirúrgica e recomendações para melhorar a qualidade da assistência na cesárea. Para concluir, foram encontradas poucas publicações com alto nível de evidência, havendo a necessidade da realização de mais estudos, notadamente por enfermeiros.

DESCRITORES: Qualidade da Assistência à Saúde; Segurança do Paciente; Cesárea; Lista de Checagem.

\section{SURGICAL SAFETY IN CESAREAN SECTION: INTEGRATIVE REVIEW}

\begin{abstract}
Our goal was to gather and analyze scientific publications on surgical safety in cesarean deliveries through the following question: What is the scientific evidence for surgical safety in cesarean deliveries in the last 10 years? An integrative review of the literature was carried out in seven electronic databases between October and December 2014. We consulted the US National Library of Medicine, the Cumulative Index of Nursing and Allied Health Literature, Latin American and Caribbean Health Sciences Literature, the Medical Literature analysis and Retrieval System Online, the Cochrane Library, and the Nursing Database and Scientific Electronic Library Online. Four hundred and sixty-three articles were found, of which 11 were selected for analysis, and two central themes emerged: promotion of patient safety in cesarean deliveries by means of the surgical safety checklist and recommendations to improve quality of care in cesarean deliveries. Finally, few publications with high levels of evidence were found, which suggests the need for further studies to be carried out, particularly by nurses. DESCRIPTORS: Quality of health care, Patient Safety, Cesarean Delivery, Checklist.
\end{abstract}

\section{SEGURIDAD QUIRÚRGICA EN LA CESÁREA: REVISIÓN INTEGRATIVA}

RESUMEN: Estudio cuyo objetivo fue reunir y analizar publicaciones científicas acerca de la seguridad quirúrgica en la cesárea con la siguiente cuestión: ¿Cuáles son las evidencias científicas sobre seguridad quirúrgica en la cesárea en los últimos diez años? Se realizó revisión integrativa de la literatura, en siete bases electrónicas, de octubre a diciembre de 2014: The US National Library of Medicine, Cumulative Index to Nursing and Allied Health Literature, Literatura Latinoamericana y del Caribe en Ciencias de la Salud, Medical Literature Analysis and Retrievel System Online, Cochrane Library, Base de datos en Enfermería y Scientific Eletronic Library Online. Fueron encontrados 463 artículos, de los cuales 11 fueron seleccionados para análisis, resultando dos ejes temáticos: promoción de la seguridad de la paciente en la cesárea por medio de la lista de verificación de seguridad quirúrgica y recomendaciones para mejorar la cualidad de la asistencia en la cesárea. Para concluir, fueron encontradas pocas publicaciones con alto nivel de evidencia, siendo necesaria la realización de más estudios, de modo especial por enfermeros.

DESCRIPTORES: Cualidad de la Asistencia a la Salud; Seguridad del Paciente; Cesárea; Lista de Verificación. 


\section{INTRODUÇÃO}

O aprimoramento das técnicas cirúrgicas, a inovação e o desenvolvimento tecnológico têm aumentado a frequência de cesáreas em várias partes do mundo. Essa evolução permitiu que a cesárea se tornasse uma das cirurgias mais difundidas. No entanto, todas as operações cirúrgicas possuem riscos, entre esses, os que mais se destacam são hemorragia, infecção pósparto e complicações da anestesia, além de maior probabilidade de complicações em virtude de cesáreas repetidas ${ }^{(1-2)}$.

Dados nacionais indicam que, ao ano, ocorrem cerca de três milhões de nascimentos, sendo grande parte deles por meio de cesáreas. A utilização desta intervenção cirúrgica aumentou no país de 1994 a 2010. Em 1994, a taxa nacional era de $32 \%$, sendo que em 2010 alcançou 52\%. Também há uma tendência crescente em países como Holanda e Estados Unidos da América $(E U A)^{(3-5)}$. Entretanto, a cesárea implica em maior exposição a riscos inerentes à própria cirurgia.

Para minimizar riscos, tem-se discutido protocolos de segurança cirúrgica em diversos países e em todos os tipos de cirurgias. Contudo, não é mais possível obter um bom funcionamento dos serviços de saúde sem investimentos na qualidade, prevenção de danos à saúde, humanização, gestão e no tratamento dispensado a usuários e profissionais envolvidos ${ }^{(6)}$.

Nesse sentido, as discussões em torno da segurança do paciente tem assumido grande notoriedade, tendo em vista que muitos dos eventos danosos, também denominados Eventos Adversos (EA), podem ser prevenidos ou sanados por meio de condutas que garantam boas práticas pautadas em evidências científicas ${ }^{(7)}$.

A Organização Mundial de Saúde (OMS) relata que um EA é uma ocorrência advinda do cuidado que resultou em danos a um paciente. A cada ano, cerca de 234 milhões de cirurgias são realizadas no mundo e em torno de sete milhões de pacientes apresentam incidentes, dos quais dois milhões resultam em mortes, quando $50 \%$ desses eventos poderiam ser prevenidos ${ }^{(8)}$.

Em pesquisa conduzida em dois hospitais brasileiros, dos 1.103 pacientes estudados, 56 sofreram EA. As infecções associadas aos cuidados da saúde e as complicações cirúrgicas e/ou anestésicas representaram 44,6\% dos danos, considerado o maior percentual em relação a outros fatores ${ }^{(9)}$. Outro estudo conduzido em
2008 revelou que um em cada 150 pacientes hospitalizados morre em decorrência de um incidente, e que quase dois terços dos EA foram relacionados à assistência cirúrgica ${ }^{(10)}$.

Somam-se outras razões para ocorrência de efeitos adversos na cirurgia, como a precária comunicação e distração entre a equipe multiprofissional, a não conferência da identificação do paciente e de materiais durante a assistência, entre outros, que constituem indicadores importantes para elevar a propensão de $\operatorname{erros}^{(11)}$.

O desenvolvimento de uma cultura de segurança, a prática dos registros em saúde, a discussão das circunstâncias em que os EA ocorreram, assim como repensar as condutas profissionais e organizacionais frente aos incidentes, são um caminho a ser seguido para a transformação da realidade nas instituições de saúde $^{(12)}$.

Diante do exposto, há uma tendência mundial para adotar práticas baseadas em evidências científicas recomendadas internacionalmente pela OMS. Assim, as pressões para proporcionar melhorias tangíveis na segurança e qualidade têm crescido entre líderes do sistema de saúde e formuladores de políticas, que estão buscando a melhor forma de organizar os serviços e equilibrar esta demanda ${ }^{(13)}$.

A fim de adotar estratégias que envolvam a segurança do paciente na área cirúrgica, em 2008 a OMS divulgou uma Lista de Verificação de Segurança Cirúrgica (LVSC), desenvolvida dentro de padrões internacionalmente aceitos de evidência científica, podendo ser adaptada à realidade de cada serviço e em vários países ${ }^{(8)}$.

A aplicação da LVSC demonstrou resultados satisfatórios em relação à diminuição de complicações e riscos cirúrgicos. Em estudo piloto pré e pós-intervenção da OMS, utilizando a lista de verificação em oito hospitais de oito cidades do mundo, os resultados demonstraram redução das taxas de mortes e das complicações entre pacientes que se submeteram a vários tipos de cirurgia ${ }^{(14)}$.

Em 2011, o Colégio Americano de Obstetras e Ginecologistas elaborou uma lista de verificação sucinta de segurança cirúrgica para a cesárea, iniciativa que contemplou alguns itens de segurança, como por exemplo, a solicitação do consentimento informado para a cesariana, indicação e quantidade de ecografias gestacionais realizadas, entre outros itens ${ }^{(15)}$. 
Embora aparentemente simplista, a evidência suporta o fato que a paciente se beneficia de checklists bem desenhados quando utilizados de forma eficaz. Uma implementação eficiente requer treinamento e mudança na cultura de segurança, bem como o estabelecimento de uma rotina de controle, medição e feedback regular dos resultados ${ }^{(16)}$.

No Brasil, a implantação de uma adaptação da LVSC da OMS em uma maternidade trouxe benefícios significativos que resultaram na adesão da direção para estabelecer a utilização da lista como rotina mandatória nas cirurgias ginecológicas eletivas. Diante disso, foram realizadas ações de sensibilização com as equipes do centro cirúrgico, por meio de reuniões educativas, buscando prepará-las para a sua utilização ${ }^{(17)}$.

Assim, os referidos estudos evidenciam a importância da segurança cirúrgica, uma vez que essa possa oferecer benefícios potenciais para a melhoria do cuidado prestado. Nesse entendimento, surgiu o interesse em reunir evidências científicas sobre a temática segurança cirúrgica na cesárea, considerando que é uma das cirurgias mais realizadas mundialmente e que há um movimento atual de readequação dos serviços de atenção obstétrica às práticas de segurança do paciente.

Assim, objetivou-se reunir e analisar publicações científicas sobre segurança cirúrgica na cesárea com a seguinte questão norteadora: quais as evidências científicas sobre segurança cirúrgica na cesárea nos últimos dez anos?

\section{MÉTODO}

Trata-se de uma pesquisa de revisão integrativa da literatura que se propôs a investigar criteriosamente as produções científicas disponíveis sobre segurança cirúrgica na cesárea. A revisão integrativa da literatura permite a construção de uma análise ampla da literatura, contribuindo para discussões sobre métodos e resultados de pesquisa, o que facilita a incorporação de evidências que fundamentam as condutas ou tomada de decisão, proporcionando um saber crítico ${ }^{(18-19)}$.

Para realização da revisão, foram seguidas as seguintes etapas: elaboração da pergunta norteadora, busca de publicações científicas em bases eletrônicas de dados, coleta de dados, análise crítica dos estudos, discussão dos resultados e apresentação da revisão integrativa ${ }^{(19)}$.
Os critérios de inclusão foram: artigos publicados em língua portuguesa, inglesa ou espanhola, em bases de dados nacionais e internacionais, revisados por pares, que abordassem a temática segurança cirúrgica na cesárea, sem delimitação da categoria do manuscrito, disponíveis on-line e na íntegra, no período compreendido entre 2005 e 2014. Foram excluídas publicações que não se encontravam disponíveis em texto completo e/ou em idioma não definidos nos critérios de inclusão e/ou fora do período delimitado para a pesquisa e/ou que não abordavam a temática definida.

$\mathrm{Na}$ busca dos artigos foram consultadas sete bases eletrônicas de dados: The US National Library of Medicine (PubMed), Cumulative Index to Nursing and Allied Health Literature (CINAHL), Literatura Latino-Americana e do Caribe em Ciências da Saúde (LILACS), Medical Literature analysis and Retrievel System Online (MEDLINE), Cochrane Library, Base de dados em Enfermagem (BDENF), Scientific Eletronic Library Online (SciELO), nos meses de outubro, novembro e dezembro de 2014.

Os seguintes descritores e suas combinações foram utilizados empregando-se o operador booleano "and" nas línguas portuguesa, espanhola e inglesa, respectivamente: "segurança do paciente" and "cesárea" and "lista de checagem" and "qualidade da assistência à saúde"; "patient safety" and "cesarean section" and "checklist" and "quality of health care"; "seguridade del paciente" and "cesárea" and "lista de verificacíon" and "calidad de la atención de salud".

A análise e síntese dos dados extraídos dos artigos foram realizadas de forma descritiva, possibilitando observar, contar, descrever e classificar os dados, com o intuito de reunir o conhecimento produzido sobre o tema explorado na revisão ${ }^{(19)}$.

Para tratamento e categorização dos resultados, foi empregado o sistema de hierarquia dos níveis de evidências: nível I - evidências provenientes de revisão sistemática ou metanálise de ensaios clínicos randomizados controlados ou oriundos de diretrizes clínicas baseadas em revisões sistemáticas de ensaios clínicos randomizados controlados; nível II - evidências derivadas de pelo menos um ensaio clínico randomizado controlado; nível III - evidências obtidas de ensaios clínicos sem randomização; nível IV - estudos de coorte e caso-controle; nível V - revisão sistemática de estudos descritivos e qualitativos; VI - evidências derivadas de um 
estudo descritivo e/ou qualitativos; nível VII opinião de autoridades ou relatório de comitês de especialistas ${ }^{(20)}$.

\section{RESULTADOS}

$\mathrm{Na}$ pesquisa indexada em bases eletrônicas, foram encontrados 463 artigos com resumo. Após leitura criteriosa dos títulos e dos resumos dos periódicos e ao serem aplicados os critérios de inclusão e exclusão, excluíram-se 452 artigos por não estarem em conformidade com o tema, com o período delimitado, idiomas definidos e não disponibilizados na íntegra. Foram selecionadas 11 publicações, lidas detalhadamente e analisadas, conforme apresentado na Tabela 1.

A síntese dos estudos analisados é apresentada nos Quadros 1 e 2, com a análise distribuída em ordem crescente conforme o ano de publicação, utilizando-se onze variáveis que foram escolhidas de itens contidos em um instrumento validado para revisão integrativa: título, autoria, área profissional dos autores, ano, periódico, tipo do estudo, base de dados, idioma, país, resultados e nível de evidência ${ }^{(21)}$.

Conforme a análise das variáveis mencionadas, observou-se que a base eletrônica PubMed destacou-se com a maioria das publicações, no total de seis manuscritos que aderiram à temática, todos encontrados em língua inglesa e oriundos dos EUA, Inglaterra e Canadá, seguidas de uma publicação proveniente da Nigéria, uma da Holanda e outra da Espanha.

Dois artigos foram encontrados na base de dados CINAHL, em língua inglesa, um proveniente dos EUA e o outro do Reino Unido, seguidos de um artigo encontrado na biblioteca Cochrane, em língua inglesa originária do Reino Unido e um na LILACS em língua espanhola oriundo da Espanha. Vale ressaltar a ausência de publicações brasileiras sobre a temática.

Referente à área de atuação profissional dos autores, todos pertenciam à área médica, nas especialidades de obstetrícia e ginecologia, anestesiologia, cirurgia e patologia molecular. Destaca-se que não foram localizadas publicações produzidas por enfermeiros.

Quanto ao ano de publicação, constatou-se que a maioria dos artigos foi publicada nos últimos cinco anos, e apenas dois dos onze artigos foram publicados em 2006 e 2008, respectivamente.

Com relação às variáveis resultados e nível de
Tabela 1 - Publicações distribuídas por quantitativo, segundo base eletrônica consultada. Brasília, DF, Brasil, 2014

\begin{tabular}{lcc}
$\begin{array}{l}\text { Base de Dados } \\
\text { Eletrônica }\end{array}$ & $\begin{array}{c}\text { Número } \\
\text { de Artigos } \\
\text { Encontrados }\end{array}$ & $\begin{array}{c}\text { Número } \\
\text { de Artigos } \\
\text { Selecionados }\end{array}$ \\
\hline Cochrane Library & 3 & 1 \\
\hline PubMed & 348 & 6 \\
\hline SciELO & 6 & 0 \\
\hline LILACS & 34 & 1 \\
\hline MEDLINE & 49 & 1 \\
\hline CINAHL & 23 & 2 \\
\hline BDENF & 0 & 0 \\
\hline Total & 463 & 11
\end{tabular}

evidência, nota-se que cinco dos onze artigos selecionados apresentaram nível de evidência VII, ou seja, envolveu opinião de especialistas sobre o assunto e cartas ao editor, seguidas de um artigo de revisão sistemática de estudo descritivo com nível de evidência $V$, dois estudos descritivos do tipo antes e depois com nível VI de evidência, e apenas dois estudos com nível de evidência I e II, respectivamente, de estudos randomizados controlados.

Para interpretação, análise e discussão, os resultados foram divididos em dois eixos temáticos: promoção da segurança da paciente na cesárea por meio da lista de verificação de segurança cirúrgica, e recomendações para melhorar a qualidade da assistência na cesárea.

\section{DISCUSSÃO}

Promoção da segurança da paciente na cesárea por meio da lista de verificação de segurança cirúrgica

A elaboração de LVSC na obstetrícia demonstrou ser eficiente na redução de danos e EA, apontou melhoria na comunicação da equipe cirúrgica e tornou as práticas mais seguras com a adoção de intervenções baseadas cientificamente, proporcionando não somente a segurança da usuária, como também dos próprios profissionais que realizam os procedimentos ${ }^{(22-25)}$.

Em estudo conduzido por meio de um questionário aplicado aos profissionais da equipe cirúrgica e às pacientes, antes e depois da introdução da LVSC, em um centro obstétrico de um hospital no Reino Unido, os achados demonstraram significante progresso na ação integrada da equipe cirúrgica no cumprimento das etapas da cirurgia, melhorando efetivamente a comunicação interprofissional e reduzindo a 


\begin{tabular}{|c|c|c|c|}
\hline Títulos das publicações & $\begin{array}{l}\text { Autoria/Ano/ } \\
\text { Área profissional }\end{array}$ & $\begin{array}{l}\text { Periódico/ } \\
\text { Tipo do estudo }\end{array}$ & $\begin{array}{l}\text { Base de } \\
\text { dados/ } \\
\text { Idioma/ } \\
\text { País } \\
\end{array}$ \\
\hline $\begin{array}{l}\text { Um Checklist de estilo da aviação para } \\
\text { condução da cesariana sob anestesia geral é } \\
\text { uma ameaça para a segurança do paciente? }\end{array}$ & $\begin{array}{l}\text { Schwarz SKW, } \\
\text { 2006. Anestesiologia/ } \\
\text { Obstetrícia/ Ginecologia }\end{array}$ & $\begin{array}{l}\text { Anesth Analg. } \\
\text { Cartas para o } \\
\text { editor }\end{array}$ & $\begin{array}{l}\text { PubMed / } \\
\text { Inglês/ } \\
\text { Holanda }\end{array}$ \\
\hline $\begin{array}{l}\text { Resultados melhorados, cesáreas e litígios } \\
\text { reduzidos decorrentes de um novo } \\
\text { paradigma em segurança do paciente }\end{array}$ & $\begin{array}{l}\text { Clark SL et al., } 2008 . \\
\text { Obstetrícia/ Ginecologia }\end{array}$ & $\begin{array}{l}\text { Am J Obstet } \\
\text { Gynecol. Opinião } \\
\text { de especialistas }\end{array}$ & $\begin{array}{l}\text { CINAHL/ } \\
\text { Inglês/ } \\
\text { Estados } \\
\text { Unidos } \\
\end{array}$ \\
\hline $\begin{array}{l}\text { Segurança do paciente em Obstetrícia e } \\
\text { Ginecologia }\end{array}$ & $\begin{array}{l}\text { American College } \\
\text { of Obstetricians \& } \\
\text { Gynecologists, } 2009 . \\
\text { Obstetrícia/Ginecologia }\end{array}$ & $\begin{array}{l}\text { Gynecol Obstet. } \\
\text { Opinião de } \\
\text { especialistas }\end{array}$ & $\begin{array}{l}\text { PubMed / } \\
\text { Inglês/ } \\
\text { Estados } \\
\text { Unidos }\end{array}$ \\
\hline Segurança do paciente no ambiente cirúrgico & $\begin{array}{l}\text { American College } \\
\text { of Obstetricians \& } \\
\text { Gynecologists, 2010. } \\
\text { Obstetrícia/Ginecologia }\end{array}$ & $\begin{array}{l}\text { Gynecol Obstet. } \\
\text { Opinião de } \\
\text { especialistas }\end{array}$ & $\begin{array}{l}\text { PubMed / } \\
\text { Inglês/ } \\
\text { Estados } \\
\text { Unidos }\end{array}$ \\
\hline $\begin{array}{l}\text { A introdução de um Checklist de segurança } \\
\text { cirúrgica em um centro obstétrico de } \\
\text { referência }\end{array}$ & $\begin{array}{l}\text { Kearns RJ et al., } \\
2011 . \\
\text { Anestesiologia }\end{array}$ & $\begin{array}{l}\text { BMJ Qual Saf. } \\
\text { Estudo descritivo }\end{array}$ & $\begin{array}{l}\text { CINAHL/ } \\
\text { Inglês/ } \\
\text { Reino Unido }\end{array}$ \\
\hline $\begin{array}{l}\text { O uso do Checklist de segurança cirúrgica } \\
\text { da Organização Mundial de Saúde melhora a } \\
\text { comunicação entre obstetras e anestesistas? }\end{array}$ & $\begin{array}{l}\text { Mohammed A et al., } 2012 . \\
\text { Cirurgia/Obstetrícia/ } \\
\text { Ginecologia }\end{array}$ & $\begin{array}{l}\text { BJOG. } \\
\text { Estudo descritivo }\end{array}$ & $\begin{array}{l}\text { PubMed/ } \\
\text { Inglês/ } \\
\text { Inglaterra }\end{array}$ \\
\hline $\begin{array}{l}\text { Proteger a segurança do paciente em locais } \\
\text { com poucos recursos }\end{array}$ & $\begin{array}{l}\text { Galadanci HS, } 2013 . \\
\text { Obstetrícia/ ginecologia }\end{array}$ & $\begin{array}{l}\text { Best Pract Res Clin } \\
\text { Obstet Gynaecol. } \\
\text { Revisão Sistemática } \\
\end{array}$ & $\begin{array}{l}\text { PubMed/ } \\
\text { Inglês/ } \\
\text { Nigéria }\end{array}$ \\
\hline $\begin{array}{l}\text { Lista de verificação de segurança cirúrgica } \\
\text { em obstetrícia e ginecologia }\end{array}$ & $\begin{array}{l}\text { Singh SS et al., } 2013 . \\
\text { Obstetrícia/ } \\
\text { Ginecologia }\end{array}$ & $\begin{array}{l}\text { J Obstet Gynaecol } \\
\text { Can. Reflexão }\end{array}$ & $\begin{array}{l}\text { PubMed / } \\
\text { Inglês/ } \\
\text { Canadá }\end{array}$ \\
\hline $\begin{array}{l}\text { Implementação das recomendações de } \\
\text { diretrizes internacionais baseadas em } \\
\text { evidências sobre cesarianas na Holanda }\end{array}$ & $\begin{array}{l}\text { Melman et al., } \\
\text { 2013. Obstetrícia/ } \\
\text { Ginecologia }\end{array}$ & $\begin{array}{l}\text { Implement Sci. } \\
\text { Ensaio clínico } \\
\text { controlado } \\
\text { randomizado }\end{array}$ & $\begin{array}{l}\text { MEDLINE/ } \\
\text { Inglês/ } \\
\text { Holanda }\end{array}$ \\
\hline Lista de verificação para cesáreas e partos & $\begin{array}{l}\text { Rosado JP et al., } 2013 . \\
\text { Pediatria }\end{array}$ & $\begin{array}{l}\text { Medwave. Carta } \\
\text { aos editores }\end{array}$ & $\begin{array}{l}\text { LILACS/ } \\
\text { Espanhol/ } \\
\text { Espanha }\end{array}$ \\
\hline $\begin{array}{l}\text { Antibiótico profilaxia versus não profilaxia } \\
\text { para prevenção de infecção depois da } \\
\text { cesárea }\end{array}$ & $\begin{array}{l}\text { Smaill FM, Grivell } \\
\text { RM, 2014. Patologia e } \\
\text { Medicina molecular }\end{array}$ & $\begin{array}{l}\text { Cochrane Libr } \\
\text { Revisão sistemática }\end{array}$ & $\begin{array}{l}\text { Cochrane } \\
\text { Library/ } \\
\text { Inglês/ } \\
\text { Reino Unido }\end{array}$ \\
\hline
\end{tabular}

ansiedade das pacientes quanto à assistência recebida $^{(26)}$.

Da mesma forma, a adesão aos critérios de qualidade adotados em forma de protocolo em doze hospitais na Holanda revelou progresso na comunicação entre a equipe e a paciente. Adicionalmente, as medidas de segurança propostas no protocolo culminaram em recomendações mais adequadas da cesárea e, por conseguinte redução de indicações desnecessárias ${ }^{(27)}$.
A precária comunicação da equipe cirúrgica reflete certa desarticulação das atividades e constitui importante desafio às instituições de saúde para implantação da cultura de segurança ${ }^{(28)}$.

A hesitação em falar pode contribuir para erros e incidentes na área de saúde. É fundamental que os profissionais expressem suas preocupações com relação à segurança da paciente, sejam conscientes dos riscos, e assim desempenhem ações e atitudes seguras ${ }^{(29)}$.

Portanto, constatou-se que é extremamente 


\begin{tabular}{|c|c|c|}
\hline Autoria & Resultados & $\begin{array}{l}\text { Nível de } \\
\text { evidência }\end{array}$ \\
\hline Schwarz SKW & $\begin{array}{l}\text { O autor refere que em recente revisão sistemática há fortes recomendações para } \\
\text { remover drogas potencialmente perigosas, em caso de anestesia geral para a } \\
\text { cesárea, a fim de minimizar erros e danos. }\end{array}$ & VII \\
\hline Clark SL et al. & $\begin{array}{l}\text { A preocupação com a segurança do paciente, por meio da aplicação de listas de } \\
\text { verificações na área de obstetrícia, tem demonstrado resultados satisfatórios que } \\
\text { conduzem à redução de cesáreas, diminuição de processos judiciais e melhoria } \\
\text { da qualidade da assistência. }\end{array}$ & VII \\
\hline $\begin{array}{l}\text { American } \\
\text { College of } \\
\text { Obstetricians \& } \\
\text { Gynecologists }\end{array}$ & $\begin{array}{l}\text { Estabelece os objetivos para segurança do paciente, que envolve comunicação, } \\
\text { práticas de medicações seguras, criação de comitê para promover a cultura de } \\
\text { segurança e evitar erros cirúrgicos. }\end{array}$ & VII \\
\hline $\begin{array}{l}\text { American } \\
\text { College of } \\
\text { Obstetricians \& } \\
\text { Gynecologists }\end{array}$ & $\begin{array}{l}\text { É apontada a necessidade de protocolos para o parto cirúrgico, uma vez que } \\
\text { reduzem a possibilidade de erros em caso de emergências obstétricas, como } \\
\text { transfusões sanguíneas, entre outras. }\end{array}$ & VII \\
\hline Kearns et al. & $\begin{array}{l}\text { Após a introdução do checklist no centro obstétrico houve melhoras } \\
\text { significativas na comunicação da equipe e redução da ansiedade nas pacientes. }\end{array}$ & VI \\
\hline $\begin{array}{l}\text { Mohammed A } \\
\text { et al. }\end{array}$ & $\begin{array}{l}\text { Os dados da pesquisa revelaram que, após a introdução da lista de verificação } \\
\text { cirúrgica da OMS, a comunicação entre obstetras e anestesistas melhorou } \\
\text { significativamente. }\end{array}$ & VI \\
\hline Galadanci HS & $\begin{array}{l}\text { O estudo revelou que, para melhorar a segurança do paciente em países em } \\
\text { desenvolvimento, é necessário melhorar a qualidade da infraestrutura dos } \\
\text { serviços de saúde e o processo da assistência em saúde. }\end{array}$ & $\mathrm{V}$ \\
\hline Singh SS et al. & $\begin{array}{l}\text { Revelou a importância das recomendações para implementação do Checklist da } \\
\text { OMS adaptado, para todos os procedimentos na cesárea voltados para genitora e } \\
\text { recém-nascido, a fim de promover qualidade e segurança cirúrgica. }\end{array}$ & VII \\
\hline Melman et al. & $\begin{array}{l}\text { Critérios de qualidade adotados em forma de protocolo nos hospitais revelaram } \\
\text { maior segurança e uma redução de cesáreas melhorando a assistência obstétrica. }\end{array}$ & II \\
\hline Rosado, JP & $\begin{array}{l}\text { O autor propõe um modelo no formato de lista de verificação tanto para o } \\
\text { parto normal, quanto para a cesárea, uma vez que já demonstrou ser eficaz em } \\
\text { diversos estudos realizados. }\end{array}$ & VII \\
\hline $\begin{array}{l}\text { Smaill FM \& } \\
\text { Grivell RM }\end{array}$ & $\begin{array}{l}\text { O estudo demonstrou que o uso de antibióticos profilaticamente previamente à } \\
\text { cesárea reduz a incidência de infecção. }\end{array}$ & I \\
\hline
\end{tabular}

eficaz e necessário elaborar e utilizar listas de verificação da saúde materna, devendo esse procedimento ser aplicado em conformidade com as características de cada serviço de saúde ${ }^{(30)}$.

Ressalta-se que listas de verificação estão agora disponíveis para diversas situações em saúde. A evidência suporta que os pacientes se beneficiam desse instrumento quando utilizados pela equipe cirúrgica de forma consciente e eficaz. No entanto, a implementação requer treinamento com avaliação constante dos resultados ${ }^{(16)}$.

A utilização da lista de verificação cirúrgica tem sido uma ferramenta que tem demonstrado sucesso em estudos conduzidos em vários hospitais e em países com diversos contextos econômicos, porém, ainda percebem-se dificuldades na implantação, no que se refere à aceitação da equipe cirúrgica(31).

Nesse sentido, o enfermeiro como líder da unidade pode adotar essa ferramenta que trará benefícios para os profissionais e pacientes, além de encorajar a participação de todos nessa iniciativa.

\section{Recomendações para melhorar a qualidade da assistência na cesárea}

Para divulgação nos serviços de saúde do uso de protocolos, programa de segurança do paciente e da lista de verificação, é importante haver consenso entre estudiosos da área de segurança do paciente, bem como das áreas afins, uma vez que as condutas devem ser embasadas cientificamente, como nos estudos que envolvem segurança no uso de medicações e procedimentos 
apropriados na cesárea. A profilaxia de infecções para a cesárea é uma delas e já vem sendo cumprida de acordo com os protocolos existentes em diversos países ${ }^{(32)}$.

Em revisão sistemática de um estudo controlado randomizado, dois grupos de mulheres foram estudados, um que recebeu o antibiótico antes da cesárea e outro que recebeu apenas placebo. $\mathrm{O}$ resultado apontou redução significativa de infecções após a cirurgia em mulheres que usaram antibióticos, o que se configurou numa recomendação mundial para a qualidade da assistência à cesárea ${ }^{(33)}$.

Em outro estudo, foi demonstrado que há fortes indicações para remover drogas potencialmente perigosas em caso de anestesia geral para a cesariana a fim de se minimizar erros e danos ${ }^{(22)}$.

Consideram-se, também, outras preocupações que envolvem a cesárea, como identificação da paciente, planejamento para fluídos corporais e vias aéreas, planejamento para anestesia, manejo apropriado para a genitora e o recémnascido, treinamento de todos os membros da equipe cirúrgica, e materiais e equipamentos em funcionamento adequado ${ }^{(23)}$.

Em hospitais que possuíam recursos humanos e materiais adequados na Nigéria, a taxa de mortalidade materna revelou ser menor. Em contrapartida, aqueles com pouca estrutura tiveram uma taxa de mortalidade materna mais elevada. O estudo concluiu que, sem melhorias na infraestrutura das unidades de saúde, as intervenções de saúde materna não terão um impacto favorável. Assim, os países em desenvolvimento são confrontados com estes desafios, demandando destes a melhoria dos serviços ofertados à população ${ }^{(34)}$.

Os problemas relacionados à segurança cirúrgica são bem reconhecidos em países desenvolvidos e em países em desenvolvimento. Nesses últimos, são fatores contributivos para as dificuldades: a deficiente infraestrutura e a inadequação de equipamentos, suprimentos e de medicamentos; a organização incipiente dos serviços de saúde, falhas no controle de infecções, falta de treinamentos para equipe e pouco recursos financeiros destinados na atenção à saúde ${ }^{(35)}$.

Aponta-se a necessidade de protocolos para a cesárea, uma vez que reduzem a possibilidade de erros em caso de emergências obstétricas, como a necessidade de transfusões sanguíneas na assistência ao parto, entre outras. É imprescindível também por estabelecer práticas de medicações seguras e também incentivar a criação de comitês para promover a cultura de segurança nas unidades de saúde ${ }^{(36-37)}$.

Melhorar os resultados maternos e promover a qualidade da assistência com vistas à segurança cirúrgica implica não somente em ações que fortaleçam a segurança do paciente, mas na redução de EA e danos a saúde da usuária, bem como da prevenção de possíveis pendências litigiosas decorrentes de falhas da equipe cirúrgica que podem ser devidamente prevenidas ${ }^{(38)}$.

\section{CONCLUSÕES}

Promover a segurança cirúrgica na cesárea implica em obter como resultado a qualidade da assistência à saúde materna. Os estudos demonstram que todas as ações que envolvem a segurança da paciente na cesárea visam estabelecer normas, protocolos e programas que conscientizem os profissionais de saúde sobre a importância de se construir uma cultura de segurança. Tais protocolos podem vir no formato de listas de verificações de segurança cirúrgicas, adaptadas às peculiaridades da área obstétrica em diferentes cenários.

Ademais, os resultados das pesquisas analisadas indicaram mudanças significativas na área de comunicação entre os profissionais da equipe cirúrgica diante de condutas preconizadas. Acresce-se ao fato de que esses avanços reduzem incidentes na atenção obstétrica.

Outro aspecto evidenciado refere-se à escassa produção sobre o assunto em pauta, revelando, ainda, serem incipientes quanto aos níveis de evidências mais rigorosos e, sobretudo, a ausência de pesquisas conduzidas por enfermeiros.

Nesse sentido, é imprescindível que os enfermeiros, como líderes e condutores da equipe de enfermagem, contribuam de forma significativa para a realização de pesquisas sobre a temática e, assim, possam colaborar com a melhoria das ações de saúde e promover a internalização da cultura de segurança cirúrgica na cesárea para todos os envolvidos, especialmente nesse momento de transformação do Sistema Único de Saúde, tendo em vista o movimento mundial para a segurança do paciente e a qualidade dos serviços de saúde.

\section{REFERÊNCIAS}

1. Flores PL, González GJP, Trejo JF, Vega GL, Cabrera 
CEP, Campos A, et al. Risk factors in cesarean section. Ginecol Obstet Mex. [Internet] 2008; 76(7) [acesso em 02 out 2015]. Disponível: http://www.ncbi.nlm.nih. gov/pubmed/18798440

2. Wiley J, Sons INC. Debo tener una cesárea? J Midwifery Womens Health. [Internet] 2013; 58(4) [acesso em 27 Jun 2015]. Disponível: http:// onlinelibrary.wiley.com/doi/10.1111/jmwh.12048/epdf

3. Ministério da Saúde (BR). Secretaria de Vigilância em Saúde. Uma análise da situação de saúde e a vigilância da saúde da mulher. Brasília: Ministério da Saúde; 2012.

4. Betrán AP, Merialdi M, Lauer JA, Bing-Shun W, Thomas J, Van Look P, et al. Rates of caesarean section: analysis of global, regional and national estimates. Pediatric and Perinatal Epidemiology. [Internet] 2007; (21) [acesso em 10 out 2014]. Disponível: http://onlinelibrary.wiley. com/doi/10.1111/j.1365-3016.2007.00786.x/epdf

5. MacDorman M, Declercq E, Menacker F. Recent trends in cesarean delivery in the United States. Clin. perinatol. [Internet] 2011; 38(2) [acesso em 11 out 2014]. Disponível: http://www.cdc.gov/nchs/data/databriefs/ db35.pdf

6. Manzo BF, Brito MJM, Corrêa AR. Implications of hospital accreditation on the everyday lives of healthcare professionals. Rev. Esc. Enferm. USP. [Internet] 2012;46(2) [acesso em 11 out 2014]. Disponível: https://dx.doi.org/10.1590/S0080-62342012000200017

7. Vendramini RCR, da Silva EA, Ferreira KASL, Possari JF, Baia WRM. Patient safety in oncology surgery: experience of the São Paulo State Cancer Institute. Rev. Esc. Enferm. USP. [Internet] 2010; 44(3) [acesso em 15 out 2014]. Disponível: http://dx.doi.org/10.1590/ S0080-62342010000300039

8. World Health Organization (WHO). World alliance for patient safety. Guidelines for safety surgery. Geneva: World Health Organization; 2008.

9. Mendes W, Martins M, Pavão ALB, Moura MLO, Travassos C. The feature of preventable adverse events in hospitals in the State of Rio de Janeiro, Brazil. Rev Assoc Med Bras. [Internet] 2013; 59(5) [acesso em 10 out 2014]. Disponível: http://dx.doi.org/10.1016/j. ramb.2013.03.002

10. de Vries EM, Ramrattan M.A, Smorenburg SM, Gouma DJ, Boermeester MA. The incidence and nature of in-hospital adverse events: a systematic review. Qual Saf Health Care. [Internet] 2008; 17(3) [acesso em 21 nov 2014]. Disponível: https://dx.doi.org/10.1136/ qshc.2007.023622

11. Bohomol E, Tartali JAA. Adverse effects in surgical patients: knowledge of the nursing professional. Acta Paul. Enferm. [Internet] 2013; 26(4) [acesso em 21 nov 2014]. Disponível: http://dx.doi.org/10.1590/S0103-

\section{2}

12. Paranaguá TT, Bezerra AL, Silva AE, Azevedo Filho FM. Prevalence of no harm incidents and adverse events in a surgical clinic. Acta paul. Enferm. [Internet] 2013; 26(3) [acesso em 17 nov 2014]. Disponível: http:// dx.doi.org/10.1590/S0103-21002013000300009

13. Driver TH, Katz PP, Trupin L, Wachter RM. Responding to clinicians who fail to follow patient safety practices: perceptions of physicians, nurses, trainees, and patients. J Hosp Med. [Internet] 2014; 9(2) [acesso em 22 nov 2014]. Disponível: http:// onlinelibrary.wiley.com/doi/10.1002/jhm.2136/epdf

14. Haynes AB, Weiser TG, William R, Berry WR, Stuart $\mathrm{R}$, Lipsitz SC et al. A surgical safety checklist to reduce morbidity and mortality in a global population. N. Engl. J. Med.. [Internet] 2009; 360(5) [acesso em 15 dez 2014]. Disponível: https://dx.doi.org/10.1056/NEJMsa0810119

15. American College of Obstetricians and Gynecologists (ACOG). Patient safety checklist n. ${ }^{\circ}$ 3: scheduling planned cesarean delivery. Gynecol Obstet. [Internet] 2011; 118(6). [acesso em 20 ago 2015]. Disponível: https://dx.doi.org/10.1097/ AOG.0b013e31823ed20d

16. Walker A, Reshamwalla S, Wilson IH. Surgical safety checklists: do they improve outcomes? Br. J. Anaesth. [Internet] 2012; 109(1) [acesso em 20 ago 2015]. Disponível: http://bja.oxfordjournals.org/ content/109/1/47.full.pdf+html

17. De Freitas MR, Antunes AG, Lopes BNA, Fernandes FDC, Monte LDC, Gama ZASG. Assessment of adherence to the WHO surgical safety checklist in urological and gynecological surgeries at two teaching hospitals in Natal, Rio Grande do Norte State, Brazil. Cad. Saúde Pública [Internet] 2014; 30(1) [acesso em 16 dez 2014]. Disponível: http://dx.doi.org/10.1590/0102$311 X 00184612$

18. Mendes KDS, Silveira RCCP, Galvão CM. Integrative literature review: a research method to incorporate evidence in health care and nursing. Texto Contexto Enferm. [Internet] 2008; 17(4) [acesso em $13 \mathrm{dez}$ 2014]. Disponível: http://dx.doi.org/10.1590/S010407072008000400018

19. Souza MT, Silva MD, Carvalho R. Revisão integrativa: o que é e como fazer? Einstein. [Internet] 2010; 8 Suppl 1 [acesso em 11 nov 2014]. Disponível: http://www. astresmetodologias.com/material/O_que_e_RIL.pdf

20. Melnyk BM, Fineout-Overholt E. Evidence-based practice in nursing \& healthcare: a guide to best practice. 2nd ed. Philadelphia: Lamp; 2012.

21. Ursi ES, Galvão CM. Prevenção de lesões de pele no perioperatório: revisão integrativa da literatura. Rev. Latino-Am. Enferm. [Internet] 2006; 14(1) [acesso 
em 12 nov 2014]. Disponível: http://www.scielo.br/pdf/ rlae/v14n1/v14n1a17.pdf

22. Schwarz SKW. Can itens on aviation-style checklist for preparation of cesarean delivery under general anesthesia present a threat for patient safety? [Letters]. Anesth Analg. [Internet] 2006; 102(3) [acesso em 12 nov 2014]. Disponível: https://dx.doi.org/10.1213/01. ANE.0000190877.56380.FE

23. Singh SS, Mehra N, Hopkins L, Leyland N, Wolfman W, Allaire C, et al. Surgical safety checklist in obstetrics and gynaecology. J Obstet Gynaecol Can. [Internet] 2013; 35 Suppl 1 [acesso em 13 dez 2014]. Disponível: http://sogc.org/wp-content/uploads/2013/04/JOGCJan2013-CPG286-ENG-Online.pdf

24. Mohammed A, Wu J, Biggs T, Ofili-Yebovi D, Cox M, Pacquette $S$, et al. Does use of a world health organization obstetric safe surgery checklist improve communication between obstetricians and anaesthetists? A retrospective study of 389 caesarean sections. BJOG. [Internet] 2012; 120 [acesso em 13 dez 2014]. Disponível: http://onlinelibrary.wiley.com/ doi/10.1111/1471-0528.12041/epdf

25. Rosado JP. Lista de verificación para cesáreas y partos. [Carta a los editores]. Medwave. [Internet] 2013; 13(2) [acesso em 13 dez 2014]. Disponível: https:// dx.doi.org/10.5867/medwave.e5629

26. Kearns RJ, Uppal V, Bonner J, Robertson J, Daniel M, McGrady EM. The introduction of a surgical safety checklist in a tertiary referral obstetric centre. BMJ Qual Saf. [Internet] 2011; 20(9) [acesso em 13 dez 2014]. Disponível: https://dx.doi.org/10.1136/ bmjqs.2010.050179

27. Melmann, Schoorel ENC, Dirksen C, Kwee A, Smits L, De Boer F, et al. Simple: implementation of recommendations from international evidence-based guidelines on caesarean sections in the Netherlands. Protocol for a controlled before and after study. Implement Sci. [Internet] 2013; (3) [acesso em $13 \mathrm{dez}$ 2014]. Disponível: https://dx.doi.org/10.1186/17485908-8-3

28. Cauduro FLF, Sarquis LM, Sarquis LMM, Cruz EDA. Safety culture among surgical center professionals. Cogitare enferm. [Internet] 2015; 20(1) [acesso em 22 ago 2015]. Disponível: http://ojs.c3sl.ufpr.br/ojs2/ index.php/cogitare/article/view/36645/24860

29. Okuyama A, Wagner C, Bijnen B. Speaking up for patient safety by hospital-based health care professionals: a literature review. BMC Health Serv Res. [Internet] 2014; 14(61) [acesso em 23 dez 2014]. Disponível: https://dx.doi.org/10.1186/1472-6963-14-61

30. Hang D O. Evaluating of primary outcomes W.H.O surgical safety checklist 2009 application in an obstetrics and gynecology hospital of Vietnam.
Am J Infect Control. [Internet] 2012; 40(5) [acesso em 24 dez 2014]. Disponível: https://dx.doi.org/10.1016/j. ajic.2012.04.231

31. Pancieri AP, Santos BP, Avila MAG, Braga EM. Checklist de cirurgia segura: análise da segurança e comunicação das equipes de um hospital escola. Rev. Gaúcha Enferm. [Internet] 2013; 34(1) [acesso em 24 dez 2014]. Disponível: https://dx.doi.org/10.1590/ S1983-14472013000100009

32. Costantine $M$, Longo $M$, Wen $T$, Hankins GDV, Saade GR. Timing of perioperative antibiotics for cesarean section: A meta-analysis. Am J Obstet Gynecol. [Internet] 2007; 197(6) [acesso em $24 \mathrm{dez}$ 2014]. Disponível: https://dx.doi.org/10.1016/j. ajog.2007.10.250

33. Smaill FM, Grivell RM, Antibiotic prophylaxis versus no prophylaxis for preventing infection after cesarean section (Cochrane Review). The Cochrane Library. [Internet] 2014; 10 [acesso em 24 dez 2014]. Disponível: http://onlinelibrary.wiley.com/doi/10.1002/14651858. CD007482.pub3/epdf.

34. Galadanci HS. Protecting patient safety in resourcepoor settings. Best Pract Res Clin Obstet Gynaecol. [Internet] 2013; 27(4) [acesso em 12 nov 2014]. Disponível: http://www.sciencedirect.com/science/ article/pii/S1521693413000370

35. World Health Organization (WHO). More than words. Conceptual framework for the international classification for patient safety Version 1.1. Geneva: World Health Organization; 2009.

36. American College of Obstetricians and Gynecologists (ACOG). Committee Opinion. No 447 Patient Safety in Obstetrics and Gynecology. Obstet Gynecol. [Internet] 2009; 114 [acesso em 12 nov 2014]. Disponível: https://dx.doi.org/10.1097/ AOG.0b013e3181c6f90e

37. American CollegeofObstetriciansandGynecologists (ACOG). Committee opinion No.464. Patient Safety in the Surgical Environment. Obstet Gynecol. [Internet] 2010; 116 [acesso em 12 nov 2014]. Disponível: https:// dx.doi.org/10.1097/AOG.0b013e3181f69b22

38. Clark SL, Belfort MA, Byrum SL, Meyers JA, Perlin JB. Improved outcomes, fewer caesarean deliveries, and reduced litigation: results of a new paradigma in patient safety. Am J Obstet Gynecol. [Internet] 2008; 199 [acesso em 12 nov 2014]. Disponível: https://dx.doi. org/10.1016/j.ajog.2008.02.031 